\title{
Evidências de Eficácia e o Excesso de Confiança Translacional da Análise do Comportamento Clínica
}

\author{
Jan Luiz Leonardi ${ }^{1}$ \\ Paradigma Centro de Ciências e Tecnologia do Comportamento, São Paulo, SP, Brasil \\ Sonia Beatriz Meyer \\ Departamento de Psicologia Clínica da Universidade de São Paulo, São Paulo, SP, Brasil
}

\begin{abstract}
Resumo
O surgimento das terapias comportamentais seguiu a mesma estratégia que havia se mostrado bem-sucedida em outras ciências: extrapolar princípios validados empiricamente na pesquisa básica para a resolução de problemas humanos. Entretanto, apesar da relevância desses princípios para a prática terapêutica, a mera transposição direta desse conhecimento para os diversos tipos de problemas clínicos não garante, a priori, a efetividade da intervenção. Tendo em vista que uma das características definidoras da Análise do Comportamento Aplicada é o forte comprometimento com a sustentação empírica de seus procedimentos de intervenção, o objetivo deste artigo é avaliar o status atual das evidências de eficácia de um ramo da Análise do Comportamento Aplicada - a Análise do Comportamento Clínica ou Terapia Analítico-Comportamental - e fazer uma reflexão crítica sobre a concepção comumente disseminada de que essas terapias são cientificamente embasadas. Para cumprir esse objetivo, o artigo apresenta um esclarecimento acerca dos termos Terapia Comportamental, Análise do Comportamento Clínica e Terapia Analítico-Comportamental, sintetiza as evidências empíricas sobre as diversas modalidades de terapia que são abarcadas pela Análise do Comportamento Clínica e argumenta que a área sofre de um "excesso de confiança translacional" (a crença de que os dados da ciência básica são suficientes para fundamentar procedimentos de intervenção).
\end{abstract}

Palavras-chave: Terapia comportamental, análise do comportamento, psicologia clínica, psicoterapia, prática baseada em evidências.

\section{Evidence of Efficacy and Translational Overconfidence in Clinical Behavioral Analysis}

\begin{abstract}
The emergence of behavioral therapies followed the same strategy that had proved successful in other fields of science: extrapolating principles, which were empirically validated in basic research, to the solution of human problems. However, the mere transposition of this knowledge to different types of clinical problems - despite the importance of these principles for therapeutic practice - does not guarantee a priori effectiveness of the intervention. One of the defining characteristics of applied behavior analysis is the strong commitment to the empirical basis of its intervention procedures. That
\end{abstract}

1 Endereço para correspondência: Rua Cardoso de Almeida, 977, Apto. 151, Perdizes, São Paulo, SP, Brasil 05013-001. E-mail: janleonardi@gmail.com

Este artigo é parte da tese de doutorado do primeiro autor (bolsista Coordenação de Aperfeiçoamento de Pessoal de Nível Superior - CAPES, processo 13.1.00056.47.7), orientada pela segunda autora (bolsista de produtividade em pesquisa do Conselho Nacional de Desenvolvimento Científico e Tecnológico - CNPq Nível 2, processo 303716/2012. 
being said, the purpose of this article is to assess the current status of the evidence of efficacy in a branch of applied behavior analysis - clinical behavior analysis or behavior-analytic therapy - and to offer a critical reflection on the commonly widespread view that these therapies are scientifically sound. To accomplish these goals, this paper clarifies terms such as Behavior Therapy, Clinical Behavior Analysis and Behavior-Analytic Therapy, summarizes the empirical evidence for the different types of therapies that are encompassed by clinical behavior analysis, and puts forth the argument that the field suffers from "translational overconfidence" (the belief that data from basic science are sufficient to support intervention procedures).

Keywords: Behavior therapy, behavior analysis, clinical psychology, psychotherapy, evidence-based practice.

\section{Las Evidencias de la Eficacia y el Exceso de Confianza Traslacional del Análisis de Conducta Clínico}

\section{Resumen}

La aparición de las terapias conductistas siguió la estrategia que había dado buenos resultados en otras ciencias: extrapolar principios validados empíricamente en la investigación básica para resolver problemas humanos. A pesar de la importancia de estos principios para la práctica terapéutica, la mera transposición directa de este conocimiento a los problemas clínicos no garantiza, a priori, la eficacia de la intervención. Sabiendo que una de las características del análisis aplicado del comportamiento es el compromiso con el apoyo empírico de sus procedimientos de intervención, el objetivo de este artículo es evaluar la situación actual de las evidencias de eficacia de una rama del Análisis Aplicado del Comportamiento - el Análisis de Conducta Clínico o Terapia Analítica-Conductual - y reflexionar de manera crítica sobre la vista generalizada de que estas terapias son científicamente sólidas. Para lograr este objetivo, el artículo presenta una aclaración de los términos Terapia Conductista, Análisis Clínico de Comportamiento y Terapia Analítica-Conductual, resume la evidencia sobre los diferentes tipos de terapia que están abarcados por el análisis clínico del comportamiento, y argumenta que el área sufre un "exceso de confianza traslacional" (la creencia de que los datos de la ciencia básica son suficientes para apoyar los procedimientos de intervención).

Palabras clave: Terapia conductista, análisis de la conducta, psicología clínica, psicoterapia, práctica basada en la evidencias.

O desenvolvimento do modelo comportamental de intervenção clínica seguiu a mesma estratégia que havia se mostrado bem-sucedida em outras ciências: extrapolar princípios validados empiricamente na pesquisa básica para a resolução de problemas aplicados. Assim, da mesma forma que a Medicina se baseava em Fisiologia e Microbiologia e a Engenharia em Física e Química, a tese defendida pelos pioneiros da terapia comportamental (e.g., Watson, Jones, Lazarus e Wolpe) era a de que qualquer intervenção deveria partir do entendimento científico dos processos comportamentais básicos, o que representou uma forte oposição à Psicologia
Clínica vigente no início do século XX (terapias psicanalíticas e humanistas em sua maioria; Branch \& Hackenberg, 1998; O’Donohue, 1998).

Já na década de 1920 foram realizadas as primeiras tentativas de transposição do conhecimento acerca dos princípios de condicionamento respondente, investigados em animais de laboratório por Pavlov no início do século, para a análise de fenômenos clínicos. Alguns exemplos são a demonstração de como a reação de medo poderia ser construída via condicionamento respondente (Watson \& Rayner, 1920) e desconstruída por meio da exposição gradual 
ao estímulo temido (Jones, 1924a, 1924b). Nas décadas de 1950 e 1960, as descobertas de Skinner (1938/1991) sobre os processos básicos do comportamento operante (e.g., reforçamento) em animais de laboratório foram estendidas para o comportamento humano. Inicialmente, os pesquisadores tinham como único objetivo avaliar se tais processos serviriam também para explicar e modificar o comportamento humano e, posteriormente, o interesse tornou-se explicitamente terapêutico, dando origem à Análise do Comportamento Aplicada (Kazdin, 1978; Moskorz, Kubo, Luca, \& Botomé, 2012; para uma exposição introdutória e didática da história das terapias comportamentais, ver Leonardi, 2015).

Assim, é possível constatar que a Análise do Comportamento Aplicada apresenta, desde a sua origem, um forte comprometimento com a sustentação empírica de seus procedimentos terapêuticos. Nesse sentido, a Declaração Sobre o Direito ao Tratamento Comportamental Eficaz, desenvolvida por uma força-tarefa da Association for Behavior Analysis (Van Houten et al., 1988), afirma que:

Um indivíduo tem direito a um tratamento eficaz e cientificamente validado. Por sua vez, os analistas do comportamento têm a obrigação de usar apenas as técnicas que a pesquisa demonstrou ser eficaz, informar os consumidores e o público sobre as vantagens e desvantagens dessas técnicas e buscar continuamente pelas melhores estratégias de mudar o comportamento. (p. 383)

De modo semelhante, as Diretrizes para Atuação Responsável de Analistas do Comportamento elaboradas pela Behavior Analyst Certification Board (2010), organização que regula a certificação profissional de analistas do comportamento em diversos países, afirma repetidamente que a prestação de serviços em Análise do Comportamento Aplicada deve ser embasada na melhor evidência disponível, como ilustram os trechos a seguir:

1.01 Respaldo no Conhecimento Científico. Analistas do comportamento se baseiam em conhecimentos derivados científica e profissionalmente ao fazer juízos científicos ou profissionais na prestação de serviços hu- manos ou quando engajados em atividades acadêmicas ou profissionais. (p. 1)

2.10 Eficácia do Tratamento. (a) O analista do comportamento sempre tem a responsabilidade de recomendar os mais eficazes procedimentos de tratamento cientificamente sustentados. Os procedimentos de tratamento eficazes são aqueles que foram validados como tendo benefícios tanto de longo prazo quanto de curto prazo para os clientes e para a sociedade. (b) Os clientes têm direito a um tratamento eficaz (i.e., baseado na literatura de pesquisa e adaptado para o cliente individual). (p. 6)

Assim, a premissa da Análise do Comportamento Aplicada parece estar em perfeita harmonia com o movimento da Prática Baseada em Evidências em Psicologia, que é, atualmente, um dos assuntos mais discutidos no cenário internacional da Psicologia (para uma apresentação histórica acerca das diversas perspectivas teóricas, conceituais e metodológicas sobre esse tema, ver Leonardi \& Meyer, 2015). Entretanto, ainda que a Análise do Comportamento Aplicada tenha como pressuposto a necessidade de sustentação empírica de seus procedimentos, é de fundamental importância avaliar em que medida a área está ou não produzindo evidências de eficácia de seus procedimentos.

Em vista disso, o presente artigo tem por objetivo avaliar o status atual das evidências de eficácia de um ramo da Análise do Comportamento Aplicada - a Análise do Comportamento Clínica ou Terapia Analítico-Comportamental e fazer uma reflexão crítica sobre a concepção comumente disseminada de que essas terapias são cientificamente embasadas. Para cumprir esse objetivo, o artigo apresenta um esclarecimento acerca dos termos Terapia Comportamental, Análise do Comportamento Clínica e Terapia Analítico-Comportamental, sintetiza as evidências empíricas sobre as diversas modalidades de terapia que são abarcadas pela Análise do Comportamento Clínica e argumenta que a área sofre de um "excesso de confiança translacional" (uma crença de que os dados da pesquisa básica são suficientes para fundamentar procedimentos de intervenção). 


\section{A Análise do Comportamento Clínica e a Terapia Analítico-Comportamental}

Desde que a expressão Terapia Comportamental foi utilizada pela primeira vez na década de 1950, uma grande diversidade de práticas foram agrupadas sob esse rótulo sem que houvesse, muitas vezes, qualquer afinidade filosófica, conceitual ou metodológica entre elas (Moskorz et al., 2012; O’Donohue, 1998).

Com o intuito de delimitar a terapia comportamental embasada nos fundamentos filosóficos, conceituais, metodológicos e empíricos da Análise do Comportamento de todo o rol de terapias comportamentais existentes, alguns autores (e.g., Kohlenberg, Hayes, \& Tsai, 1993; Kohlenberg, Tsai, \& Dougher, 1993) cunharam o termo Análise do Comportamento Clínica (CBA, do inglês Clinical Behavior Analysis). A CBA foi definida como um ramo da Análise do Comportamento Aplicada que se caracteriza pelos tipos de clientes atendidos (adultos verbalmente competentes), problemas clínicos abordados (transtornos psiquiátricos, estresse, obesidade, tabagismo, dificuldades do dia-a-dia, problemas conjugais, melhora da qualidade de vida, etc.), procedimentos utilizados (interações verbais entre terapeuta e cliente) e ambiente no qual a intervenção é conduzida (consultório; Guinther \& Dougher, 2013). Contudo, a CBA não é constituída de um único modelo terapêutico, mas abarca diversas terapias, tais como Terapia de Aceitação e Compromisso (ACT, do original Acceptance and Commitment Therapy), Psicoterapia Analítica-Funcional (FAP, do original Functional Analytic Psychotherapy), Terapia Comportamental Dialética (DBT, do original Dialectical Behavior Therapy), Ativação Comportamental (BA, do original Behavioral Activation), entre outras (Pérez-Álvarez, 2006).

No Brasil, o desenvolvimento de uma terapia embasada nos fundamentos da Análise do Comportamento seguiu um percurso diferente do ocorrido nos EUA (cf. Leonardi, 2015). Aqui, os princípios básicos aprendidos em livros-texto de Análise do Comportamento (e.g., Keller \& Schoenfeld, 1950/1995) e nas pesquisas publi- cadas no Journal of the Experimental Analysis of Behavior, além da abordagem Skinneriana ao comportamento verbal e à subjetividade, foram transpostos para o âmbito da psicoterapia (Guilhardi, 2003; Vandenberghe, 2011). Bellodi (2011), ao entrevistar diversos pioneiros da terapia comportamental no Brasil, constatou que um modelo de intervenção foi sendo gradualmente elaborado por eles, uma vez que, à época (década de 1970), os analistas do comportamento estrangeiros atuavam com populações severamente incapacitadas (e.g., autistas e psicóticos) em instituições e a prática clínica em consultório nos EUA era dominada por terapeutas cognitivos.

Ao longo das décadas de 1970 e os anos 2000, diversas expressões foram empregadas para se referir a essa prática clínica. Alguns exemplos são Psicoterapia Comportamental (e.g., Lettner \& Rangé, 1988), Terapia Comportamental (e.g., Guedes, 1993) e Psicologia Clínica Comportamental (e.g., Silvares, 2000/2012). Em 2001, Tourinho e Cavalcante propuseram o uso do termo Terapia Analítico-Comportamental (TAC), que se tornou consenso entre terapeutas de diferentes regiões do Brasil como a melhor denominação para qualificar sua prática profissional, por especificar, já em seu nome, as bases filosóficas, conceituais e metodológicas que a sustentam. Deve-se ressaltar que a criação do termo não teve a intenção de propor uma nova modalidade de psicoterapia, mas apenas uniformizar o nome da prática clínica fundamentada na ciência do comportamento Skinneriana que vinha sendo praticada no Brasil desde o início da década de 1970 (Zamignani, Silva, \& Meyer, 2008). Enfim, como resume Vandenberghe (2011), o termo Terapia Analitico-Comportamental se refere a uma abordagem clínica fundamentada na Análise do Comportamento originada e desenvolvida no Brasil.

Definir a CBA e a TAC como terapias fundamentadas na Análise do Comportamento poderia sugerir, à primeira vista, que os diferentes modelos terapêuticos encerrados sob esses rótulos são compatíveis entre si e que todas elas são embasadas nos fundamentos filosóficos, conceituais e metodológicos da Análise do Comportamento 
(para uma apresentação detalhada desses fundamentos, ver Moore, 2008). Todavia, a relação entre elas não é tão simples assim. Nem todos os modelos terapêuticos da CBA (FAP, ACT, BA e DBT) parecem compatíveis com a Análise do Comportamento (e até entre si), sobretudo em razão do aparente desprendimento dos procedimentos terapêuticos com os princípios básicos (reforçamento, discriminação, etc.) e de um linguajar que soa mentalista para a maioria dos analistas do comportamento que valorizam o jargão Skinneriano (Vandenberghe, 2011). Entretanto, fazer uma análise crítica acerca da compatibilidade de cada um desses modelos terapêuticos com os fundamentos da Análise do Comportamento está fora do escopo do presente artigo. A despeito disso, serão apresentadas, a seguir, as evidências empíricas de eficácia que existem sobre FAP, ACT, DBT, BA e TAC.

\section{Evidências Empíricas de Eficácia da Análise do Comportamento Clínica e da Terapia Analítico-Comportamental}

Inicialmente, é importante observar que existe controvérsia na literatura sobre o que constituem evidências de boa qualidade, quais métodos devem ser empregados para estabelecer a eficácia de uma terapia, quais as melhores formas de medir os resultados e como as evidências produzidas podem ser aplicadas na prática do psicólogo clínico (Reed, Kihlstrom, \& Messer, 2006). Descrever detalhadamente cada um desses diferentes métodos de pesquisa e tipos de medidas de resultado e apontar as vantagens e desvantagens deles está foro do escopo do presente artigo. Contudo, uma breve explicação será apresentada a seguir para o leitor que não está familiarizado com metodologia de pesquisa clínica.

Os métodos de pesquisa considerados apropriados para investigar a eficácia das terapias são o ensaio clínico randomizado, o experimento de caso único e estudo de caso. O ensaio clínico randomizado é um delineamento experimental de grupo utilizado na pesquisa farmacológica que é empregado no campo da psicoterapia des- de a década de 1960. Ele consiste na seleção de uma grande amostra de participantes com um mesmo diagnóstico alocados randomicamente em um grupo experimental (no qual o tratamento em questão está presente) ou em um grupo controle (em que o tratamento em questão está ausente). Para avaliar os resultados da exposição à intervenção em questão, uma ou mais medidas quantitativas (em geral, escalas padronizadas de autorrelato, ver adiante) são realizadas com cada um dos participantes. Por fim, os dados obtidos são sumarizados por meio de testes estatísticos, o que torna possível afirmar se, na média, determinado tratamento é eficaz para certo transtorno psiquiátrico (Kendall, Comer, \& Chow, 2013). A despeito de suas limitações (cf. Borkovec \& Castonguay, 2006; Starcevic, 2003), o ensaio clínico randomizado possibilita estabelecer relações causais com bastante precisão e, por isso, tem sido considerado o padrão-ouro para determinar a eficácia das psicoterapias.

O experimento de caso único submete um mesmo indivíduo a todas as condições da pesquisa e, em vez de ter seu desempenho comparado ao de um grupo, ele serve como seu próprio controle. $\mathrm{O}$ experimento é divido em duas condições: na linha de base, a variável dependente (um ou mais comportamentos-alvo, escores em questionários de autorrelato, etc.) é mensurada repetidas vezes para obter-se o desempenho-padrão do indivíduo na ausência da intervenção, condição análoga ao grupo-controle do ensaio clínico randomizado. Na condição de tratamento, a variável independente (uma ou mais ações terapêuticas) é introduzida e novas medidas da variável dependente são realizadas (Barlow, Nock, \& Hersen, 2008; Hurst \& Nelson-Gray, 2006). Dessa forma, a eficácia de uma intervenção pode ser estabelecida por meio da mensuração continuada do desempenho do indivíduo antes, durante e após introduzir e remover a intervenção.

O estudo de caso é a narrativa fiel, sistemática e longitudinal de um processo terapêutico, geralmente baseado na memória do terapeuta, que abrange a história de vida do cliente, as intervenções realizadas, as mudanças observadas e outras informações relevantes que possam levar a uma 
melhor compreensão do caso atendido. Dessa forma, o estudo de caso não visa investigar o efeito de uma variável específica ou testar hipóteses preestabelecidas, mas descrever de forma cuidadosa e detalhada um processo terapêutico único em toda a sua complexidade (Serralta, $\mathrm{Nu}$ nes, \& Eizirik, 2011; Stiles, 2006). É importante observar que o estudo de caso não é um método experimental e, por essa razão, tem pouco valor como evidência científica para a sustentação de uma prática terapêutica.

A produção de evidências empíricas de eficácia acerca de uma terapia, seja por meio de ensaios clínicos randomizados, experimentos de caso único ou estudos de caso, requer algum tipo de medida que represente o estado clínico do cliente antes e depois do processo terapêutico (Ogles, Lambert, \& Fields, 2002). Diversas modalidades de mensuração de resultado podem ser utilizadas na pesquisa e prática da psicoterapia, tais como: questionários padronizados de autorrelato, nos quais o cliente responde a um conjunto de questões formuladas com o intuito de obter uma amostra do seu funcionamento psicológico (e.g., Inventário de Depressão de Beck; Beck, Steer, \& Brown, 2012; Outcome Questionnaire; Lambert et al., 1996); instrumentos individualizados de autorrelato (e.g., Goal Attainment Scaling; Kiresuk, Smith, \& Cardillo, 1994); relatos de terceiros, que podem ser pessoas que convivem com o cliente (esposa, pai/mãe, filhos, colegas de trabalho, amigos) ou profissionais da saúde (acompanhante terapêutico, enfermeira, terapeuta ocupacional, médico); medidas comportamentais, nas quais o foco está naquilo que o indivíduo faz e não no que ele relata (e.g., tempo de engajamento em uma atividade, frequência de comportamentos de autolesão, quantidade de cigarros fumados, etc.); medidas de eventos fisiológicos (batimentos cardíacos, pressão arterial, volume sanguíneo, tensão muscular, respiração, condutividade da pele, hormônios, imagens cerebrais, etc.).

Após essa breve digressão sobre os diversos métodos de pesquisa em Psicologia Clínica, serão apresentadas abaixo as evidências empíricas de eficácia das diferentes modalidades de análise do comportamento clínica.
Mangabeira, Kanter e Del Prette (2012) realizaram uma revisão de literatura com o objetivo de analisar o desenvolvimento da FAP desde o seu surgimento, abarcando publicações em português, inglês e espanhol. Entre os 80 artigos localizados, apenas $42 \%$ eram pesquisas empíricas, sendo que metade destas eram estudos de caso narrativos, que, por relatarem o processo terapêutico sem descrever precisamente os procedimentos utilizados e sem empregar medidas de resultado objetivas, carecem tanto de validade interna quanto de validade externa. Além disso, os autores chamam a atenção para a escassez de experimentos de caso único e para a inexistência de ensaios clínicos randomizados, considerados o padrão-ouro da pesquisa em psicoterapia. Tendo em vista que esses autores não analisaram a eficácia obtida pela FAP nesses trabalhos, Leonardi (2016) conduziu uma revisão sistemática dos estudos empíricos sobre FAP (e TAC, ver adiante) que tiveram como clientes adultos (idade igual ou superior a 18 anos) com desenvolvimento típico e cuja intervenção ocorreu exclusivamente no ambiente de consultório. $\mathrm{O}$ autor identificou 17 relatos de caso e três experimentos de caso único, sendo que todos relataram eficácia positiva (i.e., melhora da queixa clínica). Entretanto, o resultado de oito desses 20 trabalhos (i.e., $40 \%$ ) foi avaliado apenas por meio da percepção do terapeuta e/ou do cliente acerca das mudanças ocorridas (i.e., sem nenhuma medida objetiva), o que diminui seu préstimo científico. Dessa forma, embora exista alguma evidência favorável à FAP, ela carece, até o momento, de dados empíricos rigorosos que comprovem sua eficácia.

A ACT, por outro lado, é uma das modalidades de psicoterapia mais pesquisadas no mundo. Em junho de 2015, o website da Association for Contextual Behavioral Science listava 102 ensaios clínicos randomizados para diversos quadros clínicos, como depressão, ansiedade, transtorno de personalidade borderline, transtorno obsessivo-compulsivo, tricotilomania, transtornos alimentares, abuso de substâncias, estresse,

$2 \mathrm{http} / / /$ contextualscience.org/ACT_Randomized Controlled Trials 
dor crônica, fibromialgia, obesidade, tabagismo, entre outros. Entretanto, uma revisão sistemática com meta-análise publicada recentemente (Öst, 2014) revelou que a esmagadora maioria dos ensaios clínicos randomizados sobre ACT contém sérios problemas metodológicos que invalidam seus resultados. Entre eles, destacam-se os seguintes: $51 \%$ das pesquisas utilizaram apenas um ou dois terapeutas nos grupos ACT e 76\% não avaliaram a integridade da intervenção (i.e., a aderência do terapeuta ao protocolo de intervenção) - em ambos os casos, a melhora observada comprovaria apenas que o terapeuta foi eficaz e não o protocolo utilizado; $67 \%$ das pesquisas tiveram grupos-controle com menos horas de terapia do que o grupo ACT - neste caso, a melhora observada poderia ser fruto do tempo de duração da terapia e não da especificidade da intervenção; $45 \%$ das pesquisas que revelaram superioridade da ACT em relação aos outros tipos de intervenção utilizaram testes estatísticos errados para comparar os resultados entre os grupos. Ainda que existam ensaios clínicos randomizados sobre ACT com boa qualidade metodológica, o fato de a maioria deles possuir vieses graves impossibilita concluir com precisão se e para quais tipos de problemas clínicos essa modalidade de terapia é eficaz.

A DBT, uma forma de terapia comportamental desenvolvida para o tratamento do transtorno de personalidade borderline, dispõe de 20 ensaios clínicos randomizados publicados, de acordo com website 3 do Lineham Institute. Recentemente, a DBT foi reconhecida como sendo a melhor intervenção disponível para o transtorno de personalidade borderline em uma revisão sistemática com meta-análise (Stoffers et al., 2012) produzida pela Colaboração Cochrane (que possui os mais exigentes critérios de análise da qualidade de evidências empíricas), superando, inclusive, tratamentos farmacológicos. Apesar de ter sido elaborada como uma intervenção para o transtorno de personalidade borderline, a DBT vem sendo adaptada e testada empiricamente como tratamento para outros quadros

3 http://behavioraltech.org/downloads/Researchon-DBT_Summary-of-Data-to-Date.pdf clínicos, tais como dependência química (Dimeff \& Linehan, 2008) e transtornos alimentares (Bankoff, Karpel, Forbes, \& Pantalone, 2012), entre outros.

A BA, um tratamento para depressão que surgiu da análise comparativa dos componentes ativos da terapia cognitivo-comportamental, na qual foi demonstrado que apenas a parcela comportamental do tratamento sem o uso das técnicas cognitivas produzia a mesma melhora dos sintomas quanto a aplicação do pacote completo (cf. Jacobson et al., 1996), teve sua eficácia evidenciada em mais de 30 ensaios clínicos randomizados (para revisões, ver Cuijpers, van Straten, \& Warmerdam, 2007; Ekers, Richards, \& Gilbody, 2008; Mazzucchelli, Kane, \& Rees, 2009).

Por fim, as evidências empíricas da eficácia da TAC, o modelo brasileiro de Análise do Comportamento Clínica, são bastante escassas. Ao revisar 142 publicações brasileiras sobre terapia comportamental de 1949 a junho de 2001, Nolasco (2002) identificou que $86 \%$ da produção de conhecimento da área correspondia a trabalhos conceituais, o que levou o autor a concluir que os terapeutas analítico-comportamentais "têm comunicado pouco à comunidade científica os modelos de seu método de intervenção e os resultados práticos obtidos por meio deles" (p. 64). Mais recentemente, Leonardi (2016) fez uma revisão sistemática da literatura empírica sobre a produção brasileira de terapia comportamental, na qual identificou (além dos 19 trabalhos sobre FAP citados anteriormente) 34 estudos, sendo três experimentos de caso único e 31 relatos de caso. Todavia, a grande maioria desses trabalhos carece do rigor científico necessário para avaliar a eficácia de uma intervenção. Por exemplo, dentre esses 34 estudos, 22 (i.e., 64,7\%) tiveram como única medida de resultado a percepção do terapeuta e/ou do cliente, 25 (73,5\%) não realizaram follow-up e nenhum trabalho avaliou a integridade da intervenção, o que impede identificar quais procedimentos, técnicas ou estratégias (i.e., os comportamentos do terapeuta) constituíram o processo terapêutico. Tendo em vista a inexistência de ensaios clínicos randomizados, o pequeno número de experimentos de 
caso único e o alcance limitado dos estudos de caso para avaliar a relação de causa e efeito de uma intervenção, é possível afirmar que o modelo de terapia comportamental desenvolvido e praticado no Brasil não dispõe de dados cientificamente sólidos que comprovem sua eficácia. Mais do que isso, a ausência de avaliação da integridade das intervenções realizadas impede determinar com precisão quais são as características da prática do terapeuta comportamental brasileiro, identificar as semelhanças e diferenças dessa terapia com as outras modalidades de terapia comportamental e investigar se ela tem relevância clínica.

\section{O Excesso de Confiança Translacional da Análise do Comportamento Clínica e da Terapia Analítico-Comportamental}

Os dados oriundos de revisões sistemáticas da literatura apresentados acima (Bankoff et al., 2012; Cuijpers et al., 2007; Ekers et al., 2008; Leonardi, 2016; Mangabeira et al., 2012; Mazzucchelli et al., 2009; Öst, 2014; Stoffers et al., 2012) indicam que, com exceção para a DBT e $\mathrm{BA}$, não há evidências empíricas rigorosas da eficácia da FAP, ACT e TAC. Apesar de haver escassez de pesquisas clínicas e baixo rigor científico nos trabalhos que investigaram a eficácia das diversas modalidades de terapia comportamental (mais uma vez, com exceção para a DBT e BA), pesquisadores e terapeutas brasileiros e estrangeiros parecem ter bastante convicção de que a terapia (se é que é possível falar no singular) fundamentada na Análise do Comportamento é empiricamente sustentada. Um exemplo disso é a síntese que Forsyth e Hawkins (1997) fazem da opinião dos mais de vinte autores convidados para uma série especial do periódico Behavior Therapy motivada pelos trinta anos de fundação da Association for Advancement of Behavior Therapy. Segundo os autores, "há consenso de que a terapia comportamental está, via de regra, cumprindo sua promessa de elaborar intervenções originadas empiricamente, testadas empiricamente, eficazes e breves para aliviar o sofrimento humano" (p. 327). No Brasil, um po- sicionamento semelhante pode ser constatado no seguinte trecho retirado de Meyer et al. (2010): "a abordagem analítico-comportamental é empiricamente validada em laboratório e em situações aplicadas, tais como a clínica, por meio de demonstração de relações ordenadas entre comportamento e ambiente" (p. 157).

Possivelmente, a ideia de que uma terapia fundamentada na Análise do Comportamento é baseada em evidências tem origem em seu comprometimento com a pesquisa experimental em laboratório dos processos básicos constitutivos dos fenômenos comportamentais (e.g., Rimm \& Masters, 1974). Nesse sentido, Branch e Hackenberg (1998) lembram que "as origens de muitas técnicas terapêuticas empregadas por terapeutas comportamentais podem ser traçadas diretamente aos resultados de pesquisa com animais não humanos, e conceituações gerais empregadas por terapeutas comportamentais frequentemente têm suas raízes na pesquisa com animais não humanos" (p. 15). De modo semelhante, Hawkins e Forsyth (1997), ao comentarem sobre a abrangência de indivíduos, comportamentos e ambientes que os procedimentos comportamentais foram aplicados com sucesso, afirmam que "esse extraordinário alcance de efetividade é, em parte, por causa da relativa integração entre análise do comportamento aplicada e básica" (p. 12).

De acordo com Critchfield (2014), a crença de que os dados da ciência básica são suficientes para fundamentar procedimentos de intervenção é um sério problema da Análise do Comportamento - um "excesso de confiança translacional" (p. 37), em suas palavras. Um exemplo bastante ilustrativo é a concepção de que todo e qualquer uso de punição é prejudicial. Diversos autores (e.g., Guedes, 2011; Matos, 1981; Sidman, 1989/2009) asseveram que os subprodutos envolvidos em procedimentos de punição tornam injustificável qualquer defesa de seu uso. Embora seja possível citar muitos exemplos, a seguinte afirmação de Sidman (1989/2009) resume o repúdio por parte de muitos analistas do comportamento ao uso da punição: "punição é o método mais sem sentido, indesejável e mais fundamentalmente destrutivo de controle da conduta" (p. 91). 
Entretanto, para ser consistente com a prerrogativa da Análise do Comportamento de julgar a validade de uma afirmação com base em dados empíricos objetivos, a recomendação ou a rejeição do uso da punição deveria ser fundamentada em evidências em vez de constituir uma prerrogativa a priori exclusivamente ideológica. Assim, deve-se indagar: quais dados sustentam a noção de que a punição é prejudicial? Esboçando uma resposta a essa pergunta, Critchfield (2014) lembra que a pesquisa seminal sobre punição, realizada entre as décadas de 1940 e 1970, não foi projetada para solucionar questões de aplicação (os procedimentos envolviam a administração de choque elétrico em animais de laboratório em condições muito circunscritas), corroborando a análise de Cipani (2004) de que os experimentos sobre punição e os respectivos subprodutos observados diferem consideravelmente das circunstâncias cotidianas onde a punição costuma ser utilizada. Ademais, ao revisarem a literatura de pesquisa básica e aplicada da punição, Lerman e Vorndran (2002) concluíram que, mesmo na pesquisa básica, os resultados encontrados acerca da manutenção, generalização e efeitos adversos da punição são contraditórios e que "a extensão pela qual as descobertas sobre choque elétrico contingente a respostas de não humanos pode ser extrapolada para o tratamento de transtornos comportamentais em populações clínicas pode ser substancialmente restrita" (p. 456). Por conseguinte, questões como "a punição pode contribuir para a redução de comportamentos-problema?", "quais são os seus subprodutos?", "os benefícios compensam os eventuais efeitos nocivos?", "existem meios de diminuir esses efeitos nocivos?", "quais parâmetros tornam a punição mais ou menos eficiente?" precisam ser respondidas pela pesquisa empírica em contextos de aplicação. Em conclusão, prescrever ou condenar o uso da punição com base nos dados existentes atualmente seria precipitado.

$\mathrm{O}$ excesso de confiança translacional que circunscreve o tema da punição ilustra que, apesar da relevância dos dados oriundos da pesquisa básica para o desenvolvimento da TAC e da CBA, a mera transposição direta desse conhecimento para os mais diversos tipos de proble- mas clínicos não garante, a priori, a efetividade da intervenção (Eifert \& Plaud, 1998; Holden, 2007; Neno, 2005; O’Donohue \& Ferguson, 2006; Plaud, Eifert, \& Wolpe, 1998). Portanto, ainda que orientados pela pesquisa básica, os procedimentos, estratégias e técnicas requeridos para uma intervenção analítico-comportamental eficaz precisam ser formulados e testados no próprio âmbito da pesquisa clínica. As seguintes considerações de O'Donohue (1998), um ferrenho defensor da importância da pesquisa básica para a prática psicoterápica, ilustram o valor, mas não a suficiência, dos princípios elaborados em laboratório, e apontam para a necessidade de examinar empiricamente os procedimentos de intervenção:

É um ponto metodológico fundamental que a pesquisa de laboratório troca validade externa por validade interna. Protocolos de laboratório são simplificados a fim de isolar e melhorar o controle de variáveis independentes e aumentar a precisão da mensuração de variáveis dependentes. Ao fazer isso, a preparação de laboratório torna-se frequentemente idealizada e descolada dos fenômenos naturalísticos. Entretanto, uma vez que regularidades são descobertas no laboratório, o próximo passo é verificar se elas podem ser extrapoladas para variáveis relacionadas (mas não idênticas) no ambiente natural. Relações semelhantes podem ser encontradas em preparações de laboratório e fenômenos naturalísticos em fisiologia e medicina, por exemplo. (p. 11)

De modo semelhante, Plaud et al. (1998) alertam para o fato de que

pesquisadores em terapia comportamental precisarão prestar mais atenção à maneira pela qual o conhecimento científico é traduzido em algo que possa ser utilizado ou aplicado pelo praticante. Como a teoria da aprendizagem apenas fornece analogias para o delineamento de tratamento, os princípios básicos da teoria devem ser traduzidos no trabalho clínico em um conjunto secundário de princípios a partir dos quais o tratamento pode ser derivado. $\mathrm{O}$ processo pelo qual um clínico pode fazer transformações 
da teoria para prática da forma mais efetiva é um processo importante, complexo e mal compreendido e, portanto, um tópico digno de estudo mais detalhado. (p. 329)

Nessa direção, Neno (2005) defende que os analistas do comportamento clínicos integrem esforços em torno de programas de pesquisa para avaliar a eficácia de seus procedimentos, estratégias e técnicas de intervenção. Naturalmente, nenhum laboratório de pesquisa será capaz, sozinho, de produzir o corpo de evidências necessário para a sustentação empírica das terapias analítico-comportamentais.

Nesse sentido, deve-se notar que alguns grupos no Brasil já estão se dedicando à pesquisa clínica utilizando metodologias rigorosas, como o Laboratório de Terapia Comportamental da Universidade de São Paulo, liderado pela Prof. Dra. Sonia Beatriz Meyer (e.g., Oshiro, 2011; Santos, 2014), o Grupo de Processos Verbais na Interação Terapêutica Analítico-Comportamental do Centro Paradigma de Ciências e Tecnologia do Comportamento, liderado pelo Prof. Dr. Denis Roberto Zamignani (e.g., Zamignani \& Meyer, 2014), o Programa de Pós-Graduação em Psicologia da Universidade Federal do Paraná, liderado pela Prof. Dra. Jocelaine Martins da Silveira (e.g., Popovitz, 2013), e o Programa de Pós-Graduação em Psicologia do Desenvolvimento e Aprendizagem da Universidade Estadual Paulista, liderado pela Prof. Dra. Alessandra Turini Bolsoni-Silva (e.g., Rocha, 2012).

$\mathrm{O}$ esforço desses grupos de pesquisa revela que os analistas do comportamento clínicos estão abandonando seu excesso de confiança translacional nos dados da pesquisa básica e, gradualmente, buscando comprovar empiricamente a eficácia de suas intervenções, o que certamente é uma enorme e importante contribuição para o desenvolvimento da Análise do Comportamento como ciência e profissão.

\section{Referências}

Bankoff, S. M., Karpel, M. G., Forbes, H. E., \& Pantalone, D. W. (2012). A systematic review of dialectical behavior therapy for the treatment of eating disorders. Eating Disorders, 20, 196-215. doi:10.1080/10640266.2012.668478
Barlow, D. H., Nock, M. K., \& Hersen, M. (2008). Single case experimental designs: Strategies for studying behavior change ( $3^{\text {rd }}$ ed.). Boston, MA: Allyn and Bacon.

Beck, A. T., Steer, R. A., \& Brown, G. K. (2012). Manual do Inventário de Depressão de BeckBDI-II (C. Gorenstein, W. Y. Pang, I. L. Argimon, \& B. S. G. Werlang, Trads.). São Paulo, SP: Casa do Psicólogo.

Behavior Analyst Certification Board. (2010). Guidelines for responsible conduct for behavior analysts. Retrieved from http://www.bacb.com/ index.php?page $=57$

Bellodi, A. C. (2011). Terapia comportamental no Brasil: História de terapeutas (Dissertação de mestrado, Pontifícia Universidade Católica de São Paulo, SP, Brasil). Recuperado em http:// www.sapientia.pucsp.br/tde_busca/arquivo. php?codArquivo $=13053$

Borkovec, T. D., \& Castonguay, L. G. (2006). Effectiveness research. In J. C. Norcross, L. E. Beutler, \& R. F. Levant (Eds.), Evidence-based practices in mental health: Debate and dialogue on the fundamental questions (pp. 89-96). Washington, DC: American Psychological Association.

Branch, M. N., \& Hackenberg, T. D. (1998). Humans are animals, too: Connecting animal research to human behavior and cognition. In W. O'Donohue (Ed.), Learning and behavior therapy (pp. 1535). Boston, MA: Allyn and Bacon.

Cipani, E. (2004). Punishment on trial: A resource guide to child discipline. Reno, NV: Context Press.

Critchfield, T. S. (2014). Punishment: Destructive force or valuable social "adhesive"? Behavior Analysis in Practice, 7, 36-44. doi:10.1007/ s40617-014-0005-4

Cuijpers, P., van Straten, A., \& Warmerdam, L. (2007). Behavioral activation treatments of depression: A meta-analysis. Clinical Psychology Review, 27, 318-326. doi:10.1016/j. cpr.2006.11.001

Dimeff, L. A., \& Linehan, M. M. (2008). Dialectical behavior therapy for substance abusers. Addiction Science \& Clinical Practice, 4, 39-47. Retrieved from http://www.ncbi.nlm.nih.gov/pmc/ articles/PMC2797106/pdf/ascp-04-2-39.pdf

Eifert, G. H., \& Plaud, J. J. (1998). From behavior theory to behavior therapy: An overview. In J. J. Plaud \& G. H. Eifert (Eds.), From behavior 
theory to behavior therapy (pp. 1-14). Boston, MA: Allyn and Bacon.

Ekers, D., Richards, D., \& Gilbody, S. (2008). A metaanalysis of randomized trials of behavioural treatments of depression. Psychological Medicine, 38, 611-623. doi:10.1017/ S0033291707001614

Forsyth, J. P., \& Hawkins, R. P. (1997). Thirty years of behavior therapy: Promises kept, promises unfulfilled. Behavior Therapy, 28, 327-331. doi:10.1016/S0005-7894(97)80077-9

Guedes, M. L. (1993). Equívocos da terapia comportamental. Temas em Psicologia, 2, 81-85.

Guedes, M. L. (2011). Porque o controle aversivo não é uma possibilidade na clínica. Acta Comportamentalia, 19, 65-70. Recuperado em http://pepsic.bvsalud.org/pdf/actac/v19n4/a02.pdf

Guilhardi, H. J. (2003). Tudo se deve às conseqüências... Recuperado em http://www.itcrcampinas. com.br/pdf/helio/tudo_consequencias.pdf

Guinther, P. M., \& Dougher, M. J. (2013). From behavioral research to clinical therapy. In $\mathrm{G}$. J. Madden (Ed.), APA handbook of behavior analysis: Vol. 2. Translating principles into practice (pp. 3-32). Washington, DC: American Psychological Association.

Hawkins, R. P., \& Forsyth, J. P. (1997). The behavior analytic perspective: Its nature, prospects, and limitations for behavior therapy. Journal of Behavior Therapy and Experimental Psychiatry, 28, 7-16. doi:10.1016/S0005-7916(96)00039-0

Holden, B. (2007). The relationship between explanations and practice in behavior analysis: Some challenges, and possible solutions. European Journal of Behavior Analysis, 8, 65-76. Retrieved from http://www.ejoba.org/PDF/2007_1/ Holden_2007.pdf

Hurst, R. M., \& Nelson-Gray, R. (2006). Singleparticipant (S-P) design research. In J. C. Norcross, L. E. Beutler, \& R. F. Levant (Eds.), Evidence-based practices in mental health: Debate and dialogue on the fundamental questions (pp. 64-73). Washington, DC: American Psychological Association.

Jacobson, N .S., Dobson, K. S., Truax, P. A., Addis, M. E., Koerner, K., Gollan, J. K., ...Prince, S. E. (1996). A component analysis of cognitivebehavioral treatment for depression. Journal of Consulting and Clinical Psychology, 64, 295304. doi:10.1037/0022-006X.64.2.295
Jones, M. C. (1924a). A laboratory study of fear: The case of Peter. Pedagogical Seminary and Journal of Genetic Psychology, 31, 308-315. doi:10. 1080/00221325.1991.9914707

Jones, M. C. (1924b). The elimination of children's fears. Journal of Experimental Psychology, 7, 382-390. doi:10.1037/h0072283

Kazdin, A. E. (1978). History of behavior modification: Experimental foundations of contemporary research. Baltimore, MD: University Park Press.

Keller, F. S., \& Schoenfeld, W. N. (1995). Principles of psychology: A systematic text in the science of behavior. Acton, CA: Copley Publishing Group (Original work published 1950)

Kendall, P. C., Comer, J. S., \& Chow, C. (2013). The randomized controlled trial: Basics and beyond. In J. S. Comer \& P. C. Kendall (Eds.), The Oxford handbook of research strategies for clinical psychology (pp. 40-61). New York: Oxford University Press.

Kiresuk, T. J., Smith, A., \& Cardillo, J. E. (Eds.). (1994). Goal attainment scaling: Applications, theory, and measurement. Hillsdale, NJ: Erlbaum.

Kohlenberg, R. J., Hayes, S. C., \& Tsai, M. (1993). Radical behavioral psychotherapy: Two contemporary examples. Clinical Psychology Review, 13, 579-592. doi:10.1016/02727358(93)90047-P

Kohlenberg, R. J., Tsai, M., \& Dougher, M. J. (1993). The dimensions of clinical behavior analysis. The Behavior Analyst, 16, 271-282. Retrieved from http:/www.ncbi.nlm.nih.gov/pmc/articles/ PMC2733651/pdf/behavan00026-0141.pdf

Lambert, M. J., Hansen, N. B., Umphress, V., Lunnen, K., Okiishi, J., Burlingame, G., ...Reisinger, C. W. (1996). Administration and scoring manual for the Outcome Questionnaire (OQ45.2). Wilmington, DE: American Professional Credentialing Services.

Leonardi, J. L. (2015). O lugar da terapia analítico-comportamental no cenário internacional das terapias comportamentais: Um panorama histórico. Perspectivas em Análise do Comportamento, 6, 119-131. doi:10.18761/pac.2015.027

Leonardi, J. L. (2016). Prática baseada em evidências em psicologia e a eficácia da análise do comportamento clínica (Tese de doutorado, Universidade de São Paulo, SP, Brasil). 
Leonardi, J. L., \& Meyer, S. B. (2015). Prática baseada em evidências em psicologia e a história da busca pelas provas empíricas da eficácia das psicoterapias. Psicologia: Ciência e Profissão, 35(4), 1139-1156. doi:10.1590/1982370300155201

Lerman, D. C., \& Vorndran, C. M. (2002). On the status of knowledge for using punishment: Implications for treating behavior disorders. Journal of Applied Behavior Analysis, 35, 431434. doi:10.1901/jaba.2002.35-431

Lettner, H. W., \& Rangé, B. P. (Eds.). (1988). Manual de psicoterapia comportamental. São Paulo, SP: Manole.

Mangabeira, V., Kanter, J., \& Del Prette, G. (2012). Functional analytic psychotherapy (FAP): A review of publications from 1990 to 2010. International Journal of Behavioral Consultation and Therapy, 7, 78-89. doi:10.1037/h0100941

Matos, M. A. (1981). A ética do exercício de controles aversivos. Boletim de Psicologia, 33, $125-133$

Mazzucchelli, T., Kane, R., \& Rees, C. (2009). Behavioral activation treatments for adults: A meta-analysis and review. Clinical Psychology: Science and Practice, 16, 383-411. doi:10.1111/ j.1468-2850.2009.01178.x

Meyer, S. B., Del Prette, G., Zamignani, D. R., Banaco, R. A., Neno, S., \& Tourinho, E. Z. (2010). Análise do comportamento e terapia analítico-comportamental. In E. Z. Tourinho \& S. V. Luna (Eds.), Análise do comportamento: Investigações histórias, conceituais e aplicadas (pp. 153-174). São Paulo, SP: Roca.

Moore, J. (2008). Conceptual foundations of radical behaviorism. Cornwall-on-Hudson, NY: Sloan.

Moskorz, L., Kubo, O. M., Luca, G. G., \& Botomé, S. P. (2012). Um exame dos fundamentos para diferentes denominações das intervenções do analista do comportamento em contexto clínico. Acta Comportamentalia, 20, 343-365.

Neno, S. (2005). Tratamento padronizado: Condicionantes históricos, status contemporâneo e (in) compatibilidade com a terapia analitico-comportamental (Tese de doutorado, Universidade Federal do Pará, Belém, PA, Brasil).

Nolasco, N. C. (2002). A evolução do conceito de intervenção clínica comportamental conforme apresentada em artigos produzidos no Brasil: Uma revisão histórica (Dissertação de mes- trado, Pontifícia Universidade Católica de São Paulo, SP, Brasil).

O'Donohue, W. (1998). Conditioning and third-generation behavior therapy. In W. O'Donohue (Ed.), Learning and behavior therapy (pp. 1-14). Boston, MA: Allyn and Bacon.

O’Donohue, W., \& Ferguson, K. E. (2006). Evidence-based practice in psychology and behavior analysis. The Behavior Analyst Today, 7, 335350. doi: $10.1037 / \mathrm{h} 0100155$

Ogles, B. M., Lambert, M. J., \& Fields, S. A. (2002). Essentials of outcome assessment. New York: Wiley.

Oshiro, C. K. B. (2011). Delineamento experimental de caso único: A psicoterapia analítica funcional com dois clientes dificeis (Tese de doutorado, Universidade de São Paulo, SP, Brasil). Recuperado em http://www.teses.usp.br/teses/ disponiveis/47/47133/tde-04112011-113836/ publico/oshiro_do.pdf

Öst, L. G. (2014). The efficacy of acceptance and commitment therapy: An updated systematic review and meta-analysis. Behaviour Research and Therapy, 61, 105-121.

Pérez-Álvarez, M. (2006). La terapia de conducta de tercera generacíon. EduPsykhé, 5, 159-172. doi:10.1016/j.brat.2014.07.018

Plaud, J. J., Eifert, G. H., \& Wolpe, J. (1998). The role of theory in behavior therapy: Conceptual and practical conclusions. In J. J. Plaud \& G. H. Eifert (Eds.), From behavior theory to behavior therapy (pp. 320-331). Boston, MA: Allyn and Bacon.

Popovitz, J. M. B. (2013). A resposta contingente do terapeuta aos comportamentos clinicamente relevantes: Especificação e avaliação (Dissertação de mestrado, Universidade Federal do Paraná, Curitiba, PR, Brasil). Recuperado em http:// www.humanas.ufpr.br/portal/psicologiamestrado/files/2013/10/Disserta\%C3\%A7\%C3\%A3o-Juliana-Popovitz.pdf

Reed, G. M., Kihlstrom, J. F., \& Messer, S. B. (2006). What qualifies as evidence of effective practice? In J. C. Norcross, L. E. Beutler, \& R. F. Levant (Eds.), Evidence-based practices in mental health: Debate and dialogue on the fundamental questions (pp. 13-55). Washington, DC: American Psychological Association.

Rimm, D. C., \& Masters, J. C. (1974). Behavior therapy: Techniques and empirical findings. New York: Academic Press. 
Rocha, J. F. (2012). Efeitos de uma intervenção comportamental com treino de habilidades sociais para universitários com fobia social (Dissertação de mestrado, Universidade Estadual Paulista Júlio de Mesquita Filho, Bauru, SP, Brasil). Recuperado em http://wwwp.fc.unesp.br/BibliotecaVirtual/ArquivosPDF/DIS_MEST/DIS MEST20120127_ROCHA\%20JULIANA\%20 FERREIRA.pdf

Santos, V. M. C. (2014). Efeitos da sinalização de intervenções na psicoterapia analítica funcional (Tese de doutorado, Universidade de São Paulo, SP, Brasil). Recuperado em http://www. teses.usp.br/teses/disponiveis/47/47133/tde08052015-153617/publico/santos_mangabeira_corrigida.pdf

Serralta, F. B., Nunes, M. L. T., \& Eizirik, C. L. (2011). Considerações metodológicas sobre o estudo de caso na pesquisa em psicoterapia. Estudos de Psicologia (Campinas), 28, 502-510.

Sidman, M. (2009). Coerção e suas implicações (M. A. Andery \& T. M. Sério, Trads.). Campinas, SP: Livro Pleno. (Original publicado em 1989)

Silvares, E. F. M. (Ed.). (2012). Estudos de caso em psicologia clínica comportamental infantil: Vol. 1. Fundamentos conceituais, estudos grupais e estudos relativos a problemas de saúde (7. ed.). Campinas, SP: Papirus. (Original publicado em 2000)

Skinner, B. F. (1991). The behavior of organisms: An experimental analysis. Acton, CA: Copley Publishing Group. (Original work published 1938)

Starcevic, V. (2003). Psychotherapy in the era of evidence-based medicine. Australasian Psychiatry, 11, 278-281.

Stiles, W. B. (2006). Case studies. In J. C. Norcross, L. E. Beutler, \& R. F. Levant (Eds.), Evidence-based practices in mental health: Debate and dialogue on the fundamental questions (pp. 5764). Washington, DC: American Psychological Association.
Stoffers, J. M., Völlm, B. A., Rücker, G., Timmer, A., Huband, N., \& Lieb, K. (2012). Psychological therapies for people with borderline personality disorder. Cochrane Database of Systematic Reviews, 8. doi:10.1002/14651858.CD005652. pub2

Tourinho, E. Z., \& Cavalcante, S. N. (2001). Por que terapia analítico-comportamental? $A B P M C$ Contexto, 23, 10.

Van Houten, R., Axelrod, S., Bailey, J. S., Favel, J. E., Foxx, R. M., Iwata, B. A., \& Lovaas, O. I. (1988). The right to effective behavioral treatment. Journal of Applied Behavior Analysis, 21, 381-384. doi:10.1901/jaba.1988.21-381

Vandenberghe, L. (2011). Terceira onda e terapia analítico-comportamental: Um casamento acertado ou companheiros de cama estranhos? Boletim Contexto ABPMC, 34, 33-41. Recuperado em http://abpmc.org.br/arquivos/ publicacoes/14053689957147e94e.pdf

Watson, J. B., \& Rayner, R. (1920). Conditioned emotional reactions. Journal of Experimental Psychology, 3, 1-14. doi:10.1037/h0069608

Zamignani, D. R., \& Meyer, S. B. (2014). A pesquisa de processo em psicoterapia: Estudos a partir do instrumento SiMCCIT. São Paulo, SP: Paradigma.

Zamignani, D. R., Silva, A. C. P., Neto, \& Meyer, S. B. (2008). Uma aplicação dos princípios da análise do comportamento para a clínica: A terapia analítico-comportamental. Boletim Paradigma, 3, 9-16. Recuperado em http:// www.nucleoparadigma.com.br/mac/upload/ arquivo/Boletim2008.pdf

Recebido: 26/06/2015

$1^{a}$ revisão: 11/11/2015 Aceite final: 10/12/2015 